\title{
MATERIALS OF INTERNATIONAL VACTRAIN/ 3-rd SWEDISH-UKRAINIAN CONFERENCE ON CANCER DISEASES, JANUARY 16-17, 2017, STOCKHOLM, SWEDEN
}

The scientific conference "International VACTRAIN/ 3-rd Swedish-Ukrainian conference on cancer diseases", was held on January 16-17, 2017 at Karolinska Institutet, Stockholm, Sweden. These 1.5 days were filled with the lectures by the invited speakers and oral presentations selected on the basis of submitted abstracts from younger researchers. The meeting was focused on what we have learnt over the recent times, on the current situation, and on the important challenges for the future with focus on combating cancer.

The Vice-Chancellor of Karolinska Institutet Professor Karin Dahlman-Wright gave an overview on the cancer research at Karolinska Institutet. Cancer is an enemy that requires attack from all sides and with all available resources. Collaboration is always important to make big scientific breakthroughs and clearly cancer research is no exception. It is vital that scientific discoveries are translated efficiently into new treatment methods and programs, new medicines and techniques. Karolinska Institutet is at the forefront of cancer research in the world. More than half of the research at Karolinska Institutet is carried out together with an international party. As Ukraine strengthens its ties to the European Union, collaborations like this workshop are vital.

The head of R.E. Kavetsky Institute of Experimental Pathology, Oncology and Radiobiology (IEPOR), the National Academy of Sciences of Ukraine academician Vasyl Chekhun gave a brief overview on research and collaborations at IEPOR. He also mentioned that the VACTRAIN grant allow the researchers at IEPOR, especially younger generation to grow professionally. Dr. Kateryna Tyminska, representing Embassy of Ukraine in the Kingdom of Sweden stressed the importance of scientific collaboration in relationship of two countries. Plenty of the new data and ideas were presented by researchers from Ukraine, Sweden, and Poland.

Professors Klas Wiman, Sonia Lain and Galina Selivanova presented the data on the new cancer medicine, based on activation of the TP53 protein. The main topic of the lectures by Professors Lars Holmgren, Pontus Aspenström and Manuel Patarroyo was the involvement of laminins, cadherins and integrins in the structure and migration of the cancer cells. Professor Yihai Cao told about the antiangiogenic cancer therapy. Professor Boris Zhivotovsky explained the mechanism of chemoresistance of lung adenocarcinoma. Professor Ingemar Ernberg presented his concept of cancer "attractor" cells, at the edge of tumor biology, mathematical modeling and system biology.
Professors Hans-Gustaf Ljunggren and Britta Wahren described their work on anti-cancer immunotherapy, including the clinical trials. The lecture by Professor Svitlana Sidorenko was devoted to a putative target for immunotherapy, CD150.

Many interesting reports, concerning the choice of the targets for cancer therapy were presented by other Swedish and Ukrainian researchers (see selected abstracts). Cancer therapy and the problems of personalized cancer therapy were also in the spot of the interest. During conference, largely supported by the VACTRAIN of Horizon 2020 program, there were also the poster sessions, a good platform to discuss the new data critically. Over 70 researchers took part in the work of this conference. Definitely, this meeting helped to broaden the knowledge in medical science and to plan the new fruitful scientific works with the aim to combat cancer.

\section{SELECTED ABSTRACTS}

\section{ENDOPLASMIC RETICULUM STRESS AS A KEY FACTOR OF GENOME REPROGRAMMING IN CANCER CELLS}

$$
\text { O.O. Ratushna*, D.O. Minchenko, }
$$

O. O. Riabovol, O.Y. Luzina, O.H. Minchenko

Palladin Institute of Biochemistry, NAS of Ukraine, Kyiv 01601, Ukraine

${ }^{\star}$ E-mail: Oksana_Ratushna@hotmail.com

The endoplasmic reticulum (ER) stress represents the unfolded protein response to cope with the accumulation of unfolded or misfolded proteins. It is required to maintain the functional integrity of the ER, which is a dynamic intracellular organelle with exquisite sensitivity to alterations in homeostasis. The unfolded protein response is a key player in the development of different malignant tumors. Depending on the duration and severity of the ER stress, it leads to cell adaptation or demise. This stress is a fundamental phenomenon, which provides a secure protection of the cells from different environmental challenges and is transduced by three major ER resident stress sensors. Activation of these ER stress sensors leads to transcriptional reprogramming of the cells. The signaling pathways elicited by those stress sensors have connections with metabolic pathways and with other plasma membrane receptor signaling networks. As such, the ER has an essential position as a signal integrator in the cell and is instrumental in the different phases of tumor progression.

Inositol requiring enzyme 1 (IRE1) is the most conserved transducer of the unfolded protein response and produces either adaptive (preserve ER homeo- 
stasis) or death signals through both kinase and endoribonuclease, including unconventional splicing of XBP1 mRNA and regulated IRE1-dependent decay of mRNA (RIDD). Splice variant of XBP1 controls the expression of hundreds of the unfolded protein response-specific genes upon global translational repression to preserve ER homeostasis. We have shown that inhibition of IRE1 suppresses glioma cell proliferation and tumor growth by affecting the expression of genes encoding the tumor suppressors, TNF receptors and related proteins, key transcription factors and protein kinases, as well as numerous mitochondrial proteins. Moreover, inhibition of IRE1 endoribonuclease activity only has stronger effect on glial cell proliferation in vitro and also on glioma growth (in mouse orthotopic brain model) and gene expression profile. It concerns especially genes, encoding key regulatory factors, controlling cell proliferation, distinct from inhibition of both enzymatic activities of IRE1.

Furthermore, we have shown that hypoxia, which is an obligate interconnected component of malignant tumor growth, affects almost all studied genes. Thus, inhibition of IRE1 signaling network mostly modifies the expression of proliferation related genes, contributing to the rate of glioma cell proliferation. We have identified several perspective genes, whose expression was significantly changed in glioma cells with inhibited both, enzymatic activities of IRE1 and only its endoribonuclease. These genes, as well as IRE1 endoribonuclease and kinase can be perspective targets to the design the novel compounds for therapeutic strategies to manipulate levels of ER stress in diseases. A better understanding of the biological role of IRE1 signaling network is needed to build an integrated systematic view on IRE1 signaling. This will be applied to develop novel, innovative inhibitors and activators of this signaling enzyme.

\section{THE ADAPTOR PROTEIN Ruk/CIN85 PARADOXICALLY ENHANCES EMT OF TRIPLE NEGATIVE MOUSE BREAST ADENOCARCINOMA 4T1 CELLS \\ I. Horak*, D. Shytikov, D. Geraschenko, L. Knopfova, L. Borsig, L. Drobot \\ Palladin Institute of Biochemistry, NAS of Ukraine, Kyiv 01601, Ukraine \\ *E-mail: iryna.horak@gmail.com}

To coordinate cellular responses, cell surface receptors employ receptor-associated adaptor proteins that are composed exclusively of domains and motives involved in intermolecular interactions. The assembling of adaptor proteins-mediated supramolecular complexes is regulated in dynamic and selective fashion, thereby influencing processing of information through signaling networks. Adaptor protein Ruk/CIN85 consists of three $\mathrm{SH} 3$ domains, four blocks of proline-rich motives and C-terminal coiled-coil region, and acts as a transducer platform that participates in control of various physiological processes, such as apoptosis, ligand-induced endocytosis of receptor tyrosine kinases, vesicular trafficking, cell adhesion, motility, and invasion. It has been shown that high levels of Ruk/CIN85 contribute to the conversion of weakly invasive human breast adenocarcinoma MCF-7 cells into a more malignant phenotype. However, a number of issues with respect to the role of Ruk/CIN85 in breast carcinogenesis remain still open.

To establish syngeneic mouse model suitable for experiments in vivo we stably overexpressed or downregulated Ruk/CIN85 in triple negative mouse adenocarcinoma 4T1 cells (RukUp or RukDown cells, respectively). As it turned out, RukUp cells acquired a rounded shape, whereas RukDown cells had a more distinct epithelial phenotype indicating that possible EMT/MET processes occurred in these cells. To elucidate this issue, RukUp and RukDown cells were studied for their adhesive and invasive properties in vitro and also for the expression of several marker EMT-associated genes.

Adhesion assay of $4 \mathrm{~T} 1$ cells showed inverse dependence of adhesion to collagen type I and fibronectin on Ruk/CIN85 expression. We also demonstrate that overexpression of Ruk/CIN85 is associated with increased migration and invasion through Matrigel, collagen type I and fibronectin, as well as through endothelial cells layer. Importantly, Ruk/CIN85 down-regulation led to decrease in $4 \mathrm{~T} 1$ cells motility and invasiveness in vitro.

Using western-blot analysis of main EMT markers (vimentin and E-cadherin) we found high levels of vimentin and low of E-cadherin in RukUp cells, and the opposite results in RukDown cells. We used RT2 Profiler PCR array (Qiagen) for 84 EMT-associated genes in order to find additional molecules involved in Ruk/CIN85-mediated EMT. Ruk/CIN85 was found to positively regulate expression of such transcription factors as Mitf, Zeb2, Twist, Sparc, as well as osteopontin, EGFR and TGF- $\beta$. Negative effect of Ruk/ CIN85 was demonstrated for Bmp-7, Wnt, E-cadherin, FoxC, keratins genes Krt-7, Krt-14 and Krt-19, and MMP-9. These data suggest that adaptor protein Ruk/CIN85 is a critical regulatory component involved in EMT of breast cancer cells.

\section{BIOLOGICAL CHARACTERISTICS OF TUMOR CELLS AT THE DIFFERENT STAGES OF EMT UPON EXPOSURE TO ANTICANCER DRUGS AND CYTOKINES \\ N. Bezdieniezhnykh*, O. Lykhova, R. Kocherga, Yu. Kudryavets \\ R.E. Kavetsky Institute of Experimental Pathology, \\ Oncology and Radiobiology, NAS of Ukraine, Kyiv 03022, Ukraine \\ ${ }^{\star} E$-mail: cellbank@ukr.net}

Formation of a highly malignant metastatic tumor cell phenotype and resistance to anticancer drugs are associated with the implementation of epithelialmesenchymal transition (EMT). Thus, monitoring of EMT and the possible inhibition of this process could be helpful to inhibit the tumor progression. It is quite 
often that anti-tumor treatment is not effective, so new modalities and new targets to combat cancer should be developed. Established cell lines, primary cultures of malignant cells obtained from biopsies or ascites of patients with epithelial cancers (breast, colorectal and ovarian), immunohistochemistry, and statistical methods were used.

We found that the majority of the primary tumor cells (of ascitic fluids) showed expression of mesenchymal markers, namely vimentin and $\mathrm{N}$-cadherin. Upon culturing in vitro, on the adhesively active extracellular matrix, expression of mesenchymal markers, for example, the Twist and Slug transcriptional factors, was down-regulated. At the same time, a number of cells expressing the epithelial markers, namely E-cadherin, pan-keratin, etc. was increased. Moreover, the cells altered their sensitivity to anticancer drugs, despite the various mechanisms of drug action (taxanes, vinca alkaloids, antimetabolites). Modification of the extracellular matrix, i.e. growth on collagen or in spheroids, also significantly influenced EMT marker expression and sensitivity of cells to the drugs.

Next, we investigated the influence of components of the microenvironment, especially cytokines interferon, tumor necrosis factor, and interleukins on the established cell lines (MCF-7, MDA-MB-231, Colo 205, HT-29) that show different EMT profiles. We wanted to find the most effective inhibitors of the EMT process, thus, reducing the malignancy of tumor cells. The anticancer drugs, such as platinum-containing, camptothecins, and some others were also tested.

Concluding, we have observed a lability of phenotypic characteristics of the primary tumor cells, i.e. changes in morphology, expression of markers, associated with EMT, which correlated with their sensitivity to anticancer drugs. We identified a number of biologically active agents that might shift EMT to either side, thus inhibiting the malignant phenotype of tumor cells.

\section{ALTERNATIVE DIRECTION OF INHIBITION OF MALIGNANT PROPERTIES IN TUMOR CELLS IN VITRO AND IN VIVO BY GENE THERAPY WITH INF- $\beta$ GENE IN RECOMBINANT BACULOVIRUS VECTOR}

\section{O. Lykhova ${ }^{1, *}$, L. Strokovska ${ }^{2}$, O. Kovaleva',} N. Bezdieniezhnykh', N. Semesiuk', I. Adamenko', A. Vorontsova', Yu. Kudryavets'

${ }^{1} R$. E. Kavetsky Institute of Experimental Pathology, Oncology and Radiobiology, NAS of Ukraine, Kyiv 03022, Ukraine

${ }^{2}$ Institute of Molecular Biology and Genetics, NAS of Ukraine, Kyiv 03680, Ukraine

${ }^{\star} E$-mail: AlexxDNA@gmail.com

The aim of the study was to investigate the influence of recombinant baculovirus containing the interferon- $\beta$ gene (rBV/IFN) on phenotypic characteristics of tumor cells in vitro: morphology, growth, cytogenetic characteristics and expression of proteins associated with proliferative activity, cell cycle regula- tion, epithelial-mesenchymal transition (EMT), invasiveness, and the migration potential. Tumorigenicity and the metastatic potential of tumor cells after their transduction with $\mathrm{rBV} / \mathrm{IFN}$ were studied in vivo, using the mouse models.

The mouse Lewis lung carcinoma cells (LL cell line) and melanoma cells (MM-4 cell line) were used.

Transduction of melanoma cells and lung carcinoma cells with $\mathrm{rBV} / \mathrm{IFN}$ leads to reduction of the cell number, the growth rate, cell migration ability, soft agar colony formation, and also in inhibition of the ability to grow in a serum-free condition in vitro. The LL/rBV/IFN and $\mathrm{MM}-4 / \mathrm{rBV} / \mathrm{IFN}$ cells are arrested in $\mathrm{G}_{0} / \mathrm{G}_{1}$ and $S$ phases. Transduction of these cells with the IFN- $\beta$ gene was accompanied with the accumulation of apoptotic cells, an increased level of nuclear anomalies in MM-4 cells and the increased frequency of mitotic pathology in LL cells. Moreover, transduction of LL cells with rBV/ IFN causes significant genotoxic effects: the levels of chromosome aberrations were increased, as well as the frequency of appearance of nuclear protrusions, polyploidy and endoreduplication.

Also, the production of recombinant IFN- $\beta$ by MM- 4 and LL cells was accompanied by the changes in the expression of proteins associated with the cell cycle regulation, EMT, invasiveness and migration ability: expression of p19ARF and p21WAF1 was increased, and of $\mathrm{N}$-cadherin was decreased in MM-4 cells. The number of E-cadherinpositive LL cells was increased. In addition, transduction of lung carcinoma cells with rBV/IFN leads to significant inhibition of expression of Slug and Twist. These changes indicate a significant inhibition of EMT process and reversion of tumor cells to the "normallike" epithelial phenotype.

In result, transduction of LL carcinoma and MM-4 melanoma cells with rBV/IFN in vitro inhibits their ability to form solid tumors and lung metastases in the mice. Therapeutic intravenous administration of the rBV/IFN results in significant reduction of the number and volume of lung metastases in the mice.

Concluding, transduction of lung carcinoma and melanoma cells with rBV/IFN inhibits their sings of malignancy in vitro and in vivo, provides genotoxic effects in LL cells and leads to suppression of EMT in these cells.

\section{CD150/SLAMF1 AS A NEW POTENTIAL} TARGET FOR ANTI-TUMOR THERAPY

\section{S.P. Sidorenko*, O. Romanets-Korbut,}

I. Gordiienko, L. Kovalevska, L. Shlapatska

R.E. Kavetsky Institute of Experimental Pathology,

Oncology and Radiobiology, NAS of Ukraine, Kyiv 03022, Ukraine

*E-mail: svitasyd@yahoo.com

CD150/SLAMF1 is a prototype member of SLAM family within the immunoglobulin superfamily of surface receptors that are widely expressed on cells within hematopoietic system. Six of nine SLAMF receptors have a paired unique immunoreceptor tyrosine-based 
switch motif (ITSM) that serves as a docking site for SH2-containing proteins. In T and B lymphocytes, natural killer cells, macrophages and dendritic cells CD150 is a co-receptor molecule that mediates different signal transduction pathways depending on the availability of downstream signaling elements, especially, the adaptor protein SH2D1A/SAP. Due to highly glycosylated and sialylated extracellular Ig domains, CD150 is involved in homotypic interactions and could be considered as a pattern-recognizing receptor. It is a major entry receptor for several Morbilliviruses, including measles virus, and also a bacterial sensor that control the killing of Gram-negative bacteria. Functionally it serves as a bridge between innate and adaptive immunity.

More than $90 \%$ of lymphoid leukemia and lymphomas in adults have B-cell origin. Within B-cell lineage cell surface receptor CD150/SLAMF1 is broadly expressed starting from pre-B cells with up-regulation toward plasma cells. However, expression of CD150 is rather limited on the surface of malignant $B$ cells with the block of differentiation at the different stages of maturation. The high level of CD150 surface expression is observed in hairy cell leukemia, classical Hodgkin lymphoma, subtype of diffuse large B-cell lymphoma with activated B cell phenotype (ABC-DLBCL), primary cutaneous follicular centre B-cell lymphoma, and in $60 \%$ of chronic lymphocytic leukemia cases. Expression and functions of this antigen outside of the hematopoietic system were not fully explored. We found that CD150 was expressed in several tumors of ectodermal origin (e.g. squamous cell carcinoma of uterine cervix, rectum and oral cavity, basalioma), but not in their normal counterparts. Recently we found CD150 expression in malignant cells of central nervous system (CNS) tumors. Although CD150 was not found in different regions of normal brain tissues, our immunohistochemical study revealed its expression in $77.6 \%$ of human CNS tumors, including glioblastoma, anaplastic astrocytoma, diffuse astrocytoma, ependymoma, and others. CD150 was detected in the cytoplasm, but not on the cell surface of glioma cell lines, and it was colocalized with the endoplasmic reticulum and Golgi complex markers. In addition to the full length mRNA of the conventional mCD150 splice isoform, in glioma cells we found a highly expressed novel CD150 transcript (nCD150), containing an 83 bp insert. The insert is derived from a previously unrecognized exon designated Cyt-new, which is located 510 bp downstream of the transmembrane region exon, and is a specific feature of primate SLAMF1. Since CD150 is not revealed in normal brain tissues, but is expressed in $77.6 \%$ of CNS tumors, CD150 could be considered as a novel diagnostic marker for CNS tumors and a potential target for the therapy of gliomas, especially measles virus-based oncolytic therapy.

\section{CD150/SLAMF1 ANTIGEN IN MOLECULAR PATHOBIOLOGY OF CHRONIC LYMPHOCYTIC LEUKEMIA}

\section{Gordiienko*, L. Shlapatska, V. Kholodniuk,} L. Sklyarenko, S. Sidorenko

R.E. Kavetsky Institute of Experimental Pathology, Oncology and Radiobiology, NAS of Ukraine, Kyiv 03022, Ukraine

${ }^{\star}$ E-mail: imgordiienko@gmail.com

Recent finding indicate that CD150 cell surface expression can be used as a surrogate prognostic marker of chronic lymphocytic leukemia (CLL) favorable outcome. It was shown that CLL patients with high CD150 expression level on malignant B cells has longer treatment free survival and overall survival compared to patients, which lost CD150 cell surface expression. However, the mechanisms that underlie dependent on CD150 expression biological properties of CLL $B$ cells are not fully understood. Present study was focused on characterization of CD150 topology and isoforms expression, as well as study of CD150 mediated signaling pathways in CLL B cells.

For the first time, we found that in cell surface CD150 negative (CSCD150-) CLL cases CD150 antigen was expressed on the protein level and was localized in the cytoplasm. Within cytoplasm it was colocalized with markers of endoplasmic reticulum, Golgi apparatus and endosomes, but not lysosomes. Exclusive cytoplasmic CD150 expression in the part of CLL cases was not associated with endoplasmic reticulum stress or ceramide metabolism. Ligation of CD180, but not CD40 or BCR, leaded to slight CD150 up-regulation in csCD 150- CLL B cells. CD150 cell surface expression was positively correlated with expression of cell death receptor CD95, BCR negative regulator - CD22 and receptor of Toll-like family, potential prognostic marker of favorable outcome - CD180. The highest level of CD150 colocalization on the cell surface of CLLB cells was observed for CD180. We found that conventional transmembrane $\mathrm{mCD} 150$ is a predominant CD150 isoform in CLL. However, in 20\% of studied CLL cases nCD150 isoform was prevalent. Moreover, elevated mRNA expression of sCD150 isoforms was detected in all CLL cases, compared to normal B cells subsets. In csCD $150^{+}$CLL cases the basal level of tyrosine phosphorylation and phosphorylation of serine/threonine specific motifs, which are substrates for AMPK, Akt, PKA, PKC, CDK kinases, was higher compared to that in csCD150- CLL B cells. Outcome of CD150 mediated Akt and MAPK kinases phosphorylation in CLL B cells was dependent on basal phosphorylation level of these kinases and often demonstrated rapid p38MAPK phosphorylation and bimodal kinetics of Akt, ERK $1 / 2$ and JNK1/2 activation. Examining the transcription factors expression profile in CLL B cells revealed that high PU.1 protein expression level positively correlated with CD150 cell surface expression in CLL. Furthermore, CD150 signaling was involved in regulation of PU. 1 mRNA level in csCD $150^{+}$CLL cases. We also found that signals via CD150, in contrary to BCR, 
CD40 and CD180, significantly down-regulated mRNA expression levels of CCL3, CCL4 and IL-10 cytokines in CLL B cells. This may contribute to favorable clinical outcome of csCD150+ CLL cases.

Taken together, CLL cases are heterogeneous in cell surface CD150 expression as well as in CD150 isoforms expression.

\section{CHANGES IN EXPRESSION OF MIRNA-122, -200b, AND -320a AS PROGNOSTIC BIOMARKERS FOR BREAST CANCER}

\section{T. Borikun*, N. Lukianova, V. Chekhun}

R.E. Kavetsky Institute of Experimental Pathology, Oncology and Radiobiology, NAS of Ukraine, Kyiv 03022, Ukraine

*E-mail: tborikun@gmail.com

High heterogeneity and diversity of breast tumors make the molecular characterization based on new biological markers very important. Recent studies have shown that not only genes and proteins are important players in tumorigenesis, but also a new class of potential epigenetic tumor markers such as microRNAs (miRNAs). An important advantage of miRNA molecules is their stability in both plasma and tumor tissues.

The aim of the study was to identify the features of oncosuppressive miRNA-122, -200b, and -320a in breast cancer (BC) patients and to monitor the possible correlation between their expression and cancer progression.

The study was conducted using 110 tumor samples and serum of the BC patients and 14 serum samples of healthy volunteers. The levels of miRNAs were assessed using q-PCR. The expression levels of estrogen receptor, progesterone receptor, Her2/neu, Ki-67, $\mathrm{E}$-cadherin, and $\mathrm{N}$-cadherin were monitored by immunohistochemical analysis.

Each of miRNAs has a great network of targets involved is cellular processes, that makes them very promising diagnostic markers. Among variety of cancer-related miRNAs we choose several that can be used as molecular markers in BC prognosis and are responsible for migration, proliferation, epithelial-mesenchymal transition, and drug sensibility. We have found that the majority of BC tissues are characterized by a significant decrease in expression of miRNA- 122 and $-200 \mathrm{~b}$ (in $93.2 \%$ and $83.1 \%$ of cases, respectively). Down-regulation of miRNA-122, $-200 \mathrm{~b}$, and $-320 \mathrm{a}$ in $\mathrm{BC}$ tissue was associated with a higher staging, enhanced proliferation, and also with the absence of hormone receptors $(p<0.05)$. Serum levels of miRNA-122 and $-200 \mathrm{~b}$ were associated with the pathological stage $(p=0.005$ and $p=0.01$, respectively). Decrease of miRNA-320a levels in cancer tissue compared to normal samples was associated with the triple negative $\mathrm{BC}$ phenotype as well, and also with the active proliferation, hence, with cancer aggressiveness. Low levels of miRNA-320a in serum were observed in patients with lymph node metastases $(p=0.03)$.

Concluding, the established profiles of circulating and tumor miRNAs are associated with aggressive clinical course of BC. It makes the determination of expression of the miRNA-122, $-200 \mathrm{~b}$, and $-320 \mathrm{a}$ in the serum the base for development of noninvasive screening tools for $\mathrm{BC}$ prognosis.

\section{THE MRPS18-2 PROTEIN AS A PUTATIVE MARKER OF CARCINOGENESIS}

M. Mints' ${ }^{1}$, M. Mushtaq ${ }^{2}$, N. lurchenko ${ }^{3}$, L. Kovalevska ${ }^{3}$, L. Buchynska ${ }^{3}$, E. Kashuba ${ }^{2,3, *}$ 'Department of Women's and Children's Health (KBH), Karolinska Institutet, Stockholm 17176, Sweden

${ }^{2}$ Department of Microbiology, Tumor and Cell Biology (MTC), Karolinska Institutet, Box 280, Stockholm 17177, Sweden

${ }^{3} R$.E. Kavetsky Institute of Experimental Pathology,

Oncology and Radiobiology, NAS of Ukraine, Kyiv 03022, Ukraine

${ }^{\star}$ E-mail: Elena.Kashuba@ki.se

Endometrial cancer (EC) is one of the most frequent causes of cancer death among women in developed countries. Histopathological diagnosis and imaging techniques for EC are limited, thus new prognostic markers are needed to offer patients the best treatment and follow-up.

We showed that the level of mitochondrial ribosomal protein MRPS18-2 (S18-2) increased in EC compared with the normal endometrium and hyperplasia, based on a study of 42 patient biopsies. Importantly, high expression of free E2F1 in EC correlates well with high S18-2 expression. The EC cell line HEC-1-A, which overexpresses S18-2 constitutively, showed an increased proliferation capacity in vitro and in vivo (in SCID mice). Moreover, pan-keratin, $\beta$-catenin and $\mathrm{E}$-cadherin signals are diminished in these cells, compared to the parental HEC-1-A line, in contrast to vimentin signal that is increased. This may be associated with epithelial-mesenchymal transition.

We conclude that high expression of S18-2 and free E2F1, and low pan-keratin, $\beta$-catenin, and Ecadherin signals might be a good set of prognostic markers for EC.

\section{HIGH EXPRESSION LEVELS OF MRPS18-2 AND PRESENCE OF THE RB PROTEIN ARE REQUIRED FOR THE MAINTENANCE OF THE STEM CELL PHENOTYPE}

M. Mushtaq, *, L. Kovalevska ${ }^{2}$, G. Klein ${ }^{1}$, E. Kashuba ${ }^{1,2}$

${ }^{1}$ Department of Microbiology, Tumor and Cell

Biology (MTC), Karolinska Institutet, Box 280, Stockholm 17177, Sweden

${ }^{2} R$. E. Kavetsky Institute of Experimental Pathology, Oncology and Radiobiology, NAS of Ukraine, Kyiv 03022, Ukraine

${ }^{\star} E$-mail: Muhammad.Mushtaq@ki.se

We have found that mitochondrial ribosomal protein MRPS18-2 (S18-2) is involved in regulation of the RB-dependent pathway. It binds to both hypo- and hyperphosphorylated RB. The binding between RB and 
$\mathrm{S} 18-2$ is promoted when cytoplasmic $\mathrm{S} 18-2$ is targeted to the nucleus, and this disrupts the association of E2F1 with RB, as indicated by the increased level of free E2F1 in the nucleus. This presumably enhances the RB-dependent block to S-phase entry in the cell cycle. We have also found overexpression of the human S18-2 in immortalized primary rat embryonic fibroblasts (REFs) that showed properties of embryonic stem cells. Elevated expression of S18-2 in stem cells (our findings and analysis of published microarray data) raises the question of whether this protein co-operates with the RB protein in differentiation and carcinogenesis. We wanted to seek a relation between the expression of RB and S18-2 in Rb1-/- MEFs and stemness. We hypothesized that simultaneous expression of both proteins at the high levels might support stemness.

We have used transfections, inoculation into SCID mice, directed differentiation, quantitative polymerase chain reaction, immunostaining, immunohistochemistry, western blotting. We showed that S18-2 protein, together with RB, plays a crucial role in cell dedifferentiation. We have found that overexpression of S18-2 and RB is needed for maintenance of cell stemness. Such cells can differentiate into various cell lineages under certain conditions.

Concluding, the presence of RB and simultaneous expression of S18-2 at high levels are required for the cell stemness.

\section{IDENTIFICATION OF NOVEL MOLECULAR AND GENETIC MARKERS FOR EARLY DETECTION AND PROGNOSIS OF EPITHELIAL TUMORS

\author{
O. Mankovska*, V. Kashuba \\ Institute of Molecular Biology and Genetics, \\ NAS of Ukraine, Kyiv 03680, Ukraine \\ *E-mail: mankovsska@gmail.com
}

The main topic of our research is the identification of molecular and genetic markers for the early detection of epithelial tumors and prognosis of the course of disease. Early detection of carcinogenic process can lead to more successful treatment, thus, significantly increasing the chances for recovery. The investigation of non-invasive diagnostic tools, which can be used in clinical practice, is now the main focus of oncology. This problem can be solved by using of molecular nucleic acid markers that are present in biological fluids (serum, urine, semen etc).

We have developed several useful and effective approaches for investigation of molecular and genetic markers. We are studying both, genetic and epigenetic changes in cancers of different types, using a broad scale screening of their presence in clinical samples. The techniques and methods we use allow us to obtain reliable results and propose them for implementation in clinical practice.

\section{GENOTOXIC SENSITIVITY TO 4-HYDROXYESTRADIOL IN PERIPHERAL BLOOD LYMPHOCYTES OF ENDOMETRIAL CANCER PATIENTS}

\section{O. Brieieva*, I. Nesina, L. Buchynska}

R.E. Kavetsky Institute of Experimental Pathology, Oncology and Radiobiology, NAS of Ukraine, Kyiv 03022, Ukraine

${ }^{\star} E$-mail: olha.brie@gmail.com

The endometrial cancer (EC) pathogenesis to a large extent may be determined by genotoxic effects of estrogen metabolites, among which the 4-hydroxyestradiol (4OHE2) is characterized by the most prominent DNAdamaging properties. Genomic instability and sensitivity to genotoxic estrogen metabolites in cells of EC patients depend on the DNA repair efficiency.

The aim of the study was to analyze the level of DNA damage in peripheral blood lymphocytes ( $P B L$ ) of EC patients after treatment with the 4OHE2 and to evaluate the repair efficiency of induced DNA damage.

Total $33 \mathrm{EC}$ patients (mean age $60.2 \pm 1.5$ years) and 20 healthy women (mean age $55.7 \pm 3.1$ years) were included in the study. The level of DNA damage in PBL was measured, using comet assay and expressed by \% tail DNA (\% tDNA). Estradiol (E2) level in blood serum was assessed by ELISA $(\mathrm{pg} / \mathrm{ml})$.

Large variability in basal level of DNA damage was observed in PBL of EC patients (1.8-20.5\% tDNA) as well as healthy donors (1.1-9.2\% tDNA). In average, EC patients had significantly higher basal level of DNA damage $(7.9 \pm 0.9 \%$ tDNA) compared to healthy women $(3.5 \pm 0.5 \%$ tDNA) $(p<0.05)$. After incubation with 4OHE2 there was an increase in the \% tDNA in PBL of EC patients (8.0-57.7\% tDNA) as well as of healthy donors (7.9-46.8\% tDNA). However, the average level of induced DNA damage in EC patients was significantly higher $(38.9 \pm 2.7 \%$ tDNA) compared to healthy women $(25.8 \pm 2.2 \%$ tDNA) $(p<0.05)$. To investigate the association between the sensitivity of PBL to 4OHE2 and estradiol level in the blood, we measured the concentration of E2 in the blood serum. We found that the blood level of E2 was higher $(54.9 \pm 4.1 \mathrm{pg} / \mathrm{ml})$ in EC patients with high sensitivity of PBL to 4OHE2 than in patients with low sensitivity $(38.2 \pm 4.3 \mathrm{pg} / \mathrm{ml})$. The higher level of unrepaired DNA damage in PBL was observed in EC patients (18.6 $\pm 2.6 \%$ tDNA) compared to control group (13.1 \pm $1.4 \%$ tDNA). Furthermore, it was found that patients with high sensitivity of PBL to 4OHE2 had greater amount of unrepaired DNA damage $(24.1 \pm 4.4 \%$ tDNA) than women with low sensitivity (13.1 $\pm 1.8 \%$ tDNA).

These results indicate that PBL of EC patients are characterized by hypersensitivity to $4 \mathrm{OHE} 2$ and impaired DNA damage repair that is associated with hormonal status of women. It can be assumed that increased level of 4OHE2-induced genome instability may contribute to pathogenesis of EC. 